\section{The anchor effects on the judgment of loudness using reaction time as an index of loudness*}

\author{
SEIICHIRO NAMBAT, TOSHIE YOSHIKAWA, and SONOKO YASUDA \\ Osaka University, Toyonaka, Osaka, Japan
}

The present study was designed to investigate anchor effects on loudness judgments, using reaction time (RT) as an index of loudness. In Experiment 1, anchor effects were reexamined using verbal categories. Two kinds of anchor stimuli, 60- and 90-dB SPL 1,000-Hz pure tones, and four kinds of series stimuli, $60-, 70-, 80-$, and 90-dB tones, were used. In this experiment, clear anchor effects were found just the same as in our previous experiment. Experiment 2 was conducted using RT as an index of loudness with stimulus conditions similar to those in Experiment 1. The same anchor effects could be seen in this experiment too. As RT is quite free from the limitations inevitably accompanying the verbal responses, it may be concluded that the anchor effects reflect the shift in perception.

Perception is an unobservable intervening concept. We can only guess the perceptual world from relations between stimuli presented to Ss and the overt responses made by Ss to stimuli. Therefore, we are inevitably obliged to use responses as a clue to the law of perception. Consequently, the characteristics of responses might be confused with those of perception, and sometimes it happens that what we considered as the law of perception is found to be nothing but the law of response. This problem between perception and responses raised a serious dispute concerning adaptation level (AL) theory (Helson, 1964). Namely, Stevens (1958) criticized AL theory in two ways. First, he stated that, from the viewpoint of his power law, Helson's quantitative theory (so-called $J$ function) was based on logarithmic law (Fechner's law). Second, he asserted that Helson confused adaptation, based on physiological process, with relativity in judgment. Helson explained admirably the phenomena of color conversion (contrast, constancy, adaptation) by means of the difference between AL (adaptation reflectance: weighted geometric mean of reflectance of background and mean of reflectance of samples) and reflectance of samples. Stevens admitted it to be physiological adaptation, but he raised an objection to Helson's extending AL theory to a category judgment of weight. When lifting a series of weights ranging from 200 to $400 \mathrm{~g}, \mathrm{~S}$ judged a $400 \mathrm{~g}$ weight

\footnotetext{
*Portions of this paper were reported in Japanese at the Studies in the Humanities and Social Sciences, Osaka University, Faculty of General Education, 1970, Vol. XVIII.

+Address: Department of Psychology, Faculty of General Education, Osak University, 1-1, Machikaneyama, Toy onaka, Osaka, Japan.
} same weight as "medium" after lifting a 900-g anchor; it is quite doubtful if this shift in judgment is caused by the shift in perception. As Stevens says, this shift in judgment may be attributed merely to the relativity of judgment, change of modulus of judgment scale, semantic disposition, or adjustment. It may be essentially impossible to separate perception from judgment so long as relative words such as "loud-soft" are used in judgment. So, in order to get rid of the deficiency accompanying verbal categories, there have been many experiments concerning anchor effects using absolute physical magnitude. For example, Fillenbaum (1963) used category scale and direct ratio of height to base in the judgment of the slimness of rectangles and found no anchor effects when direct ratio was used. Harvey and Campbell (1963) found anchor effects in the judgment physical magnitude (ounce), but in the latter case the effects were smaller. Helson and Kozaki (1968) showed that the same anchor effects existed in direct estimation of the number of dots, as when a category scale was used. Bell and Bevan (1968) found clear anchor effects in the judgment of four factors of Gestalt. Besides, anchor effects have also been seen in many experiments where subliminal stimuli were used as anchors (Black \& Bevan, 1960; Goldstone, Goldfarb, Strong, \& Russell, 1962; Boardman \& Goldstone, 1962; Bevan \& Pritchard, 1963).

As described above, many experiments have been done in order to make clear the relation between perception and response concerning anchor effects. Though many of them deny that anchor effects are attributed merely to the semantic shift of scaling, as "very heavy," and then judged the of weight using category scale and they do not always come to the same conclusion. Originally a category scale itself, such as "loud-soft," is quite relative and it has also a lack in that the number of categories is limited. And yet, even if categories are displaced by verbal reports using physical magnitude, there still remains a problem. For example, while it is quite a difficult task for an untrained $S$ to make a judgment using physical magnitude, he may still use it as a kind of rating scale. On the other hand, there is a possibility that a well-trained $S$ may modify his judgment intentionally in order to estimate the physical value of the stimulus very accurately.

Therefore, we decided to use a new index instead of verbal reports. That is, seeing that RT changes monotonically according to the changes of the level of tones (Tachibana, Tashiro, Makino, \& Namba, 1961; Raab \& Grossberg, 1965), we selected to use RT as an index of loudness. As RT is not a verbal report, it is free from problems concerning semantic disposition and relativity, which inevitably accompany category judgment. Besides, in the case of category judgment, the number of categories is previously determined by the $E$, but $R T$ has no such limitation. And what is better, in RT there is little possibility that Ss' intentional modification might intervene, since Ss usually try to respond as quickly as possible.

Therefore, the present experiment was designed to investigate the problem between perception and response in AL theory concerning anchor effects, using RT. First, in Experiment 1 anchor effects on loudness judgments were reexamined, using verbal categories. In most of the previous experiments, including our own, anchor stimuli which were much louder or softer than series stimuli were used. In this experiment, however, stimuli with the same level of the end stimuli in series stimuli were used as the anchor. Next, Experiment 2 was conducted, using RT under just the same conditions as those of Experiment 1.

\section{EXPERIMENT 1}

In our previous experiment (Yoshikawa \& Namba, 1966; Yoshikawa, 1968), we confirmed that anchor effects were seen when loudness was judged using verbal categories. Again, in Experiment 1, we reexamined anchor effects, using verbal categories and afterward comparing that result with anchor effects when RT was used as an index of loudness.

\section{Method}

Stimuli. White noises were used as 


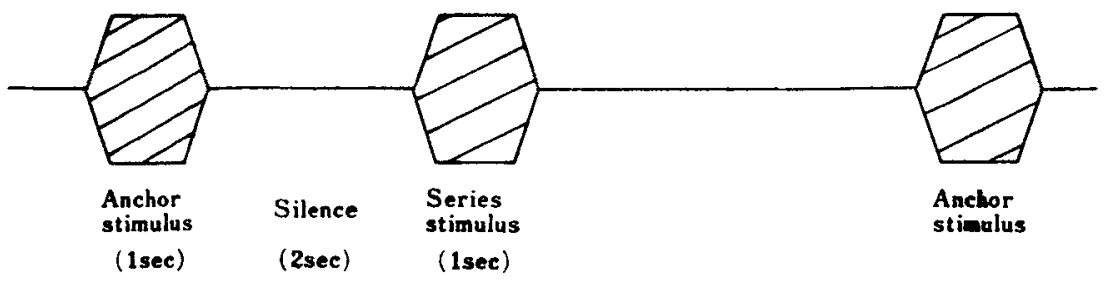

Fig. 1. Stimulus condition of Experiment 1. The stimulus duration was $1 \mathrm{sec}$, and there was a 2 -sec interval between anchor and series stimulus.

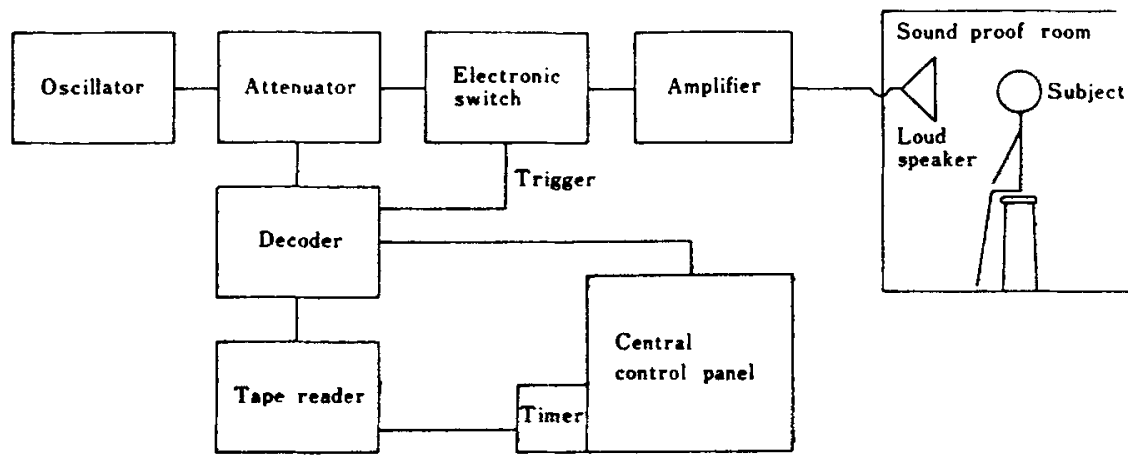

Fig. 2. Block diagram of Experiment 1.

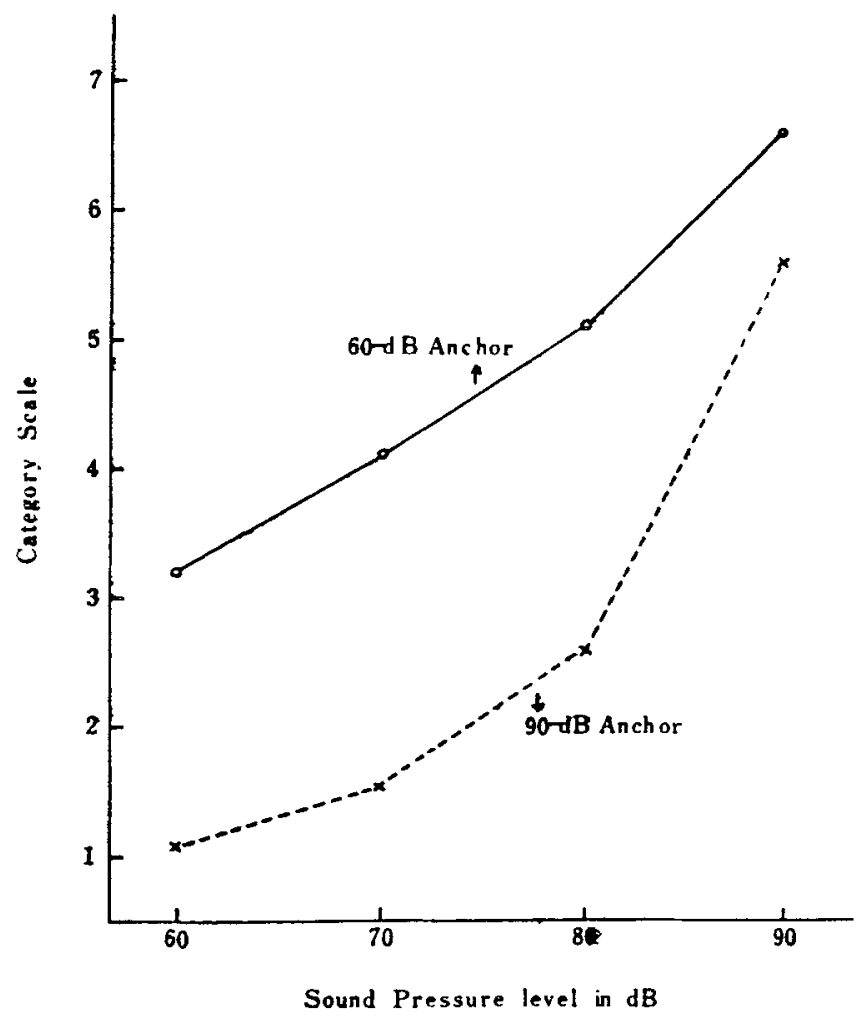

Fig. 3. Effects of anchor stimulus on the judgment of loudness when seven verbal categories were used. Each point is the mean of 100 judgments made by five Ss. Notice that stimuli were judged as much louder when a $60-\mathrm{dB}$ anchor stimulus was inserted. stimuli. The levels of the anchor stimuli were 60-dB SPL in Series 1 and 90-dB SPL in Series 2, and series stimuli were 60-, 70-, 80-, and 90-dB SPL.

Procedure. One trial consisted of a 1 -sec presentation of anchor stimulus, a 2-sec silent interval, and a 1-sec presentation of series stimulus, as illustrated in Fig. 1. Four kinds of series stimuli were presented in random order. Both in Series 1 and in Series 2, Ss were given practice twice to each of the four stimuli, and then they were given 80 trials, 20 to each stimulus. Ss judged only series stimuli, using seven categories-(1) very soft, (2) soft, (3) slightly soft, (4) medium, (5) slightly loud, (6) loud, and (7) very loud. They reported their judgments to $E$ orally during a 5 -sec interval between trials. Ss were instructed as follows: "A pair of noises will be presented to you from the loudspeaker. You have only to listen to the first noise. Please judge only the second noise absolutely using the seven categories in front of you. You must not compare it with the first one. And you need not try to judge consistently. Please report just as you perceive."

Apparatus. We used a "computer-controlled system" (Fig. 2), the details of which are described in our previous paper (Manabe, Namba, Yoshikawa, \& Kuwano, 1969). Stimuli were automatically presented to $S s$ in accordance with a previously programmed punchtape. Therefore, experimental conditions, such as stimulus levels, stimulus presentation order, stimulus presentation interval, etc., were controlled quite accurately, and $\mathrm{E}$ did not need to manipulate by himself. Noises were generated by an oscillator and their levels were measured with the $C$ network of a sound-level meter (RION NA-07A).

Subjects. Ten university students with normal hearing ability participated in this experiment. Half of them served for the experiment in Series 1 and the others in Series 2 in a soundproof room. The Ss were tested individually.

\section{Results}

Average categories of 100 judgments to each stimulus by five Ss are shown in Fig. 3. Anchor effects are clearly seen, just as in our previous experiment; when a soft anchor (60 dB) was used, judgments were shifted toward loud categories, and when a loud anchor ( $90 \mathrm{~dB})$ was used, judgments were shifted toward soft categories. For instance, an $80-\mathrm{dB}$ noise was judged as "slightly loud" (Category 5) when a soft anchor was used, while it was judged as between 
"soft" (Category 2) and "slightly soft" (Category 3) when a loud anchor was used.

\section{EXPERIMENT 2}

In Experiment 2, anchor effects were investigated using RT as an index of loudness in order to examine whether or not anchor effects resulted from the semantic shift of scaling.

\section{Method}

Stimuli. $1,000-\mathrm{Hz}$ pure tones were used as stimuli. As in Experiment 1 , the levels of anchor stimuli were $60-\mathrm{dB}$ SPL in Series 1 and 90-dB SPL in Series 2, and series stimuli were 60-, 70-, 80-, and 90-dB SPL.

Procedure. As illustrated in Fig. 4, one trial consisted of a 1-sec presentation of anchor stimulus, a silent interval (foreperiod), and a 1-sec presentation of series stimulus. Ss responded only to series stimuli by releasing the key as soon as they heard the tone. Therefore, it is quite noticeable that in this experiment the anchor stimulus acted as a warning signal for the presentation of the series stimulus, and the experimental procedure was just the same as the common experiment of simple RT. In Experiment 1, the foreperiod was kept at 2 sec. But if an anchor stimulus is presented preceding a series stimulus always by a constant interval, Ss can anticipate the presentation of the series stimulus, which is quite inconvenient for an experiment using RT. Therefore, in order to avoid the effects of Ss' expectancy, we used three different foreperiod lengths -0.5 , 2 , and $5 \mathrm{sec}$-at random. Both in series 1 and in Series 2, after preliminarily trying each of the four series stimuli, Ss were tested six times with each series stimulus. The results of the preliminary trial were omitted from data processing. Ss were instructed as follows: "Please keep the key on the table down. A pair of tones will be presented to you from the loudspeaker. You have only to listen to the first tone. And please release the key as soon as you hear the second tone. Afterward, again keep the key down."

Apparatus. The block diagram of Experiment 2 is shown in Fig. 5. Tones were generated by an oscillator, and their levels were regulated by two attenuators, one for anchor stimuli and the other for series stimuli. The tones were presented through the loudspeaker by means of an electronic switch. The electronic switch controlled both on and off time of tones. The electronic switch and the electric stopwatch were triggered by a two-circuit microswitch. That is, with a push of a trigger button, stimuli were presented to $\mathrm{Ss}$, and at the same time

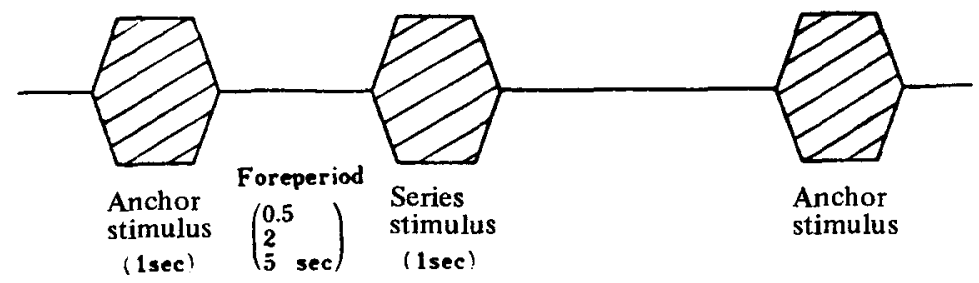

Fig. 4. Stimulus condition of Experiment 2. The duration of the stimulus was $1 \mathrm{sec}$, and three lengths of foreperiod, $0.5,2$, and $5 \mathrm{sec}$, were used randomly to avoid the effects of Ss' expectancy.

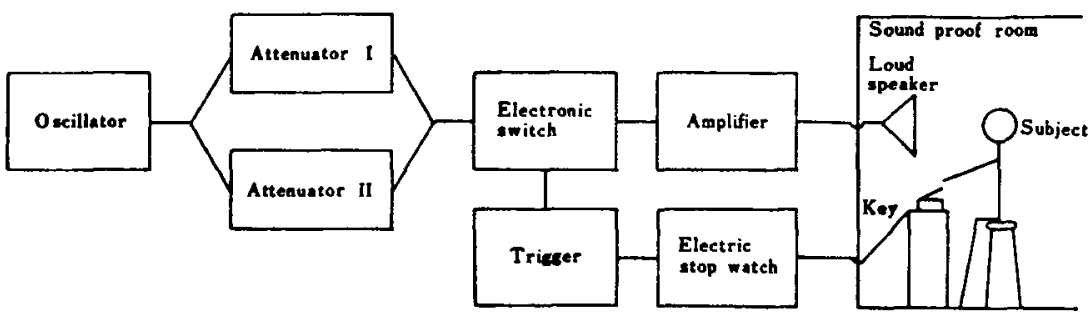

Fig. 5. Block diagram of Experiment 2.

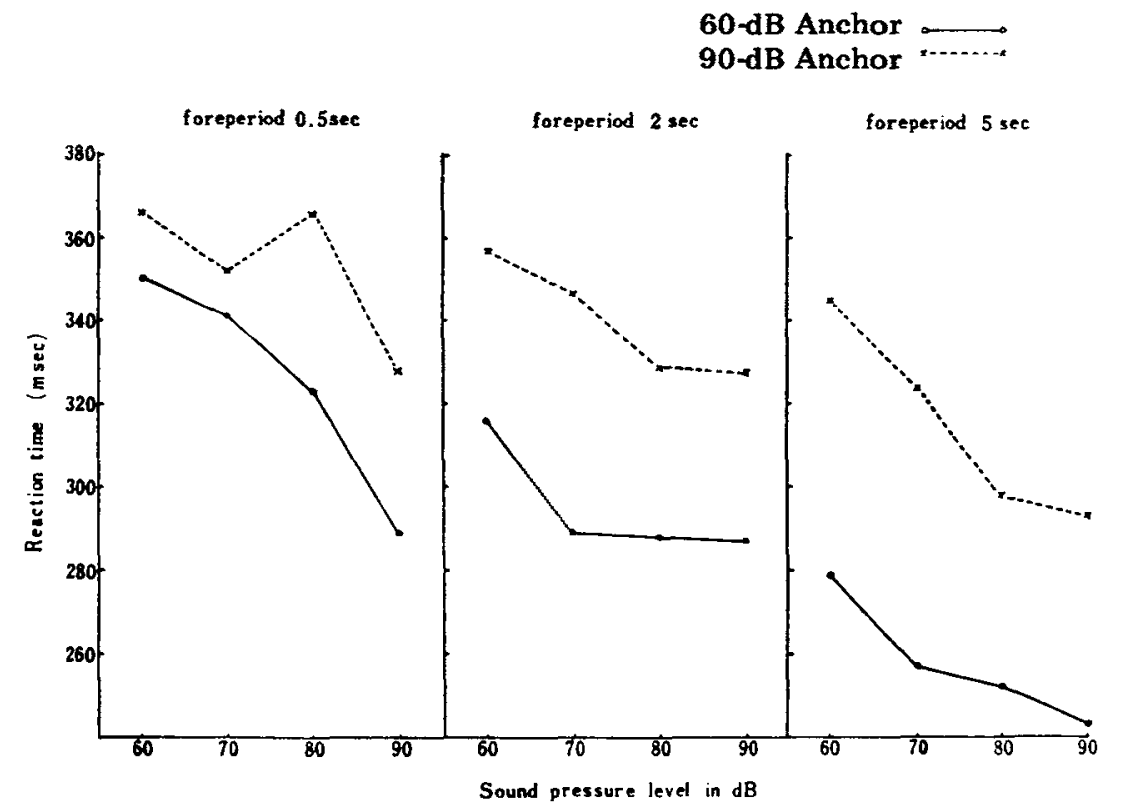

Fig. 6. Effects of anchor stimulus when RT was used. Each point is the mean of 16 responses made by eight $S$ s. It can clearly be seen in every case that $R T$ became much shorter when a 60-dB anchor stimulus was inserted.

an electric stopwatch began to move. A conventional telegraph key was put on the desk $10 \mathrm{~cm}$ in front of $\mathrm{S}$, and an electric stopwatch stopped when he released the key. E read the scale of the stopwatch and recorded it.

Subjects. Eight university students with normal hearing ability served as Ss. They were tested individually in a soundproof room. Half of them had trials of Series 1 first and then, after a 10- to 30- $\mathrm{min}$ intermission, Series 2; the order was reversed for the other half.

Results

Average RTs of 16 responses by eight $\mathrm{Ss}$ in each condition ( 2 kinds of 


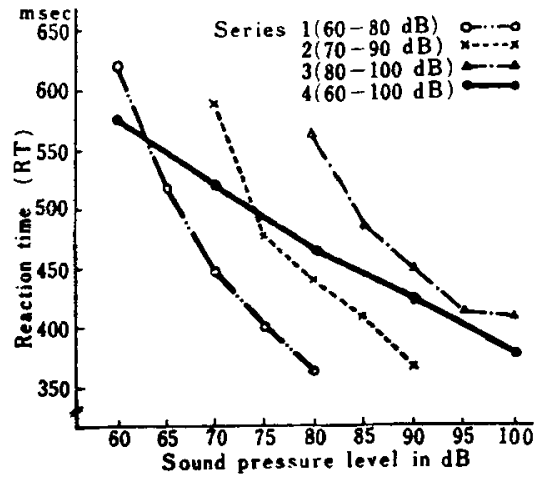

Fig. 7. RT for each timulus. Each point is the median of 100 responses made by five Ss. It is quite remarkable that there exists a context effect between sound pressure level and RT (Namba, Yoshikawa, \& Kuwano, 1968).

anchor stimulus by 4 kinds of series stimulus by 3 kinds of foreperiod) are shown in Fig. 6. As can be seen clearly, RT became shorter as the stimulus became more intense, except in one case (the 90- $\mathrm{dB}$ anchor/0.5- $\mathrm{sec}$ foreperiod/80-dB series stimulus condition). This finding coincides with previous reports concerning the relation between $R T$ and loudness and suggests that RT can be used as an index of loudness. And also the effects of foreperiod can be recognized. In any case, inspection of the results concerning anchor effects shows that RT with a 90-dB anchor are longer than RT with a 60-dB anchor in every case. Maximum difference is seen especially in RT to a 60-dB series stimulus with a 5-sec foreperiod, i.e., RT to $60 \mathrm{~dB}$ after a 90-dB anchor was $345 \mathrm{msec}$ and $R T$ to $60 \mathrm{~dB}$ after a 60-dB anchor was $279 \mathrm{msec}$. Accordingly, in the RT to tones, anchor effects were found just the same as when verbal responses were used.

\section{DISCUSSION}

The result of Experiment 1 shows clear anchor effects in judgments of loudness when verbal categories are used. As described above, in most of the previous experiments the anchor stimuli used were much larger or smaller than the series stimuli. Therefore, in such conditions, if Ss were forced to judge using verbal categories, which are quite relative and limited, they would allot a maximum category to a large anchor, even though they were not asked to judge an anchor stimulus. Consequently, series stimuli, which were smaller than the anchor stimulus, were allotted smaller categories, and judgments shifted negatively. On the other hand, when a small anchor was used, Ss would allot a minimum category to the anchor stimulus and larger categories to series stimuli. So judgments shifted positively. If this were the case, anchor effects would have no relation to Ss' perception, but would merely be the semantic shift of scaling. But in the case of Experiment 1, the levels of anchor stimuli were within the range of series stimuli. Therefore, even if categories were allotted to every stimulus, judgments would not shift as much. Consequently, anchor effects seen in Experiment 1 cannot necessarily be attributed to Ss' allotment of categories. And yet, as long as verbal responses are used, it is impossible to avoid semantic effects. So in Experiment 2 we examined anchor effects using RT instead of verbal responses; and we could see clear anchor effects just as we did category judgments in Experiment 1. RT is quite free from the relativity or limitation of numbers, etc., which inevitably accompanies verbal responses. Moreover, in Experiment 2, anchor stimuli acted as warning signals and the Ss did not notice them as an important variables of the experiment. They tried to respond to series stimuli as quickly as possible. Therefore, there is little possibility that Ss' intentional modification might intervene. Anyway, semantic factors must not intervene in this experiment. Nevertheless, anchor effects exist. So anchor effects are not always caused by the semantic shift of scaling, but occur in the shift in perception.

We have also previously done experiments using RT for context effects (Namba, Yoshikawa, \&
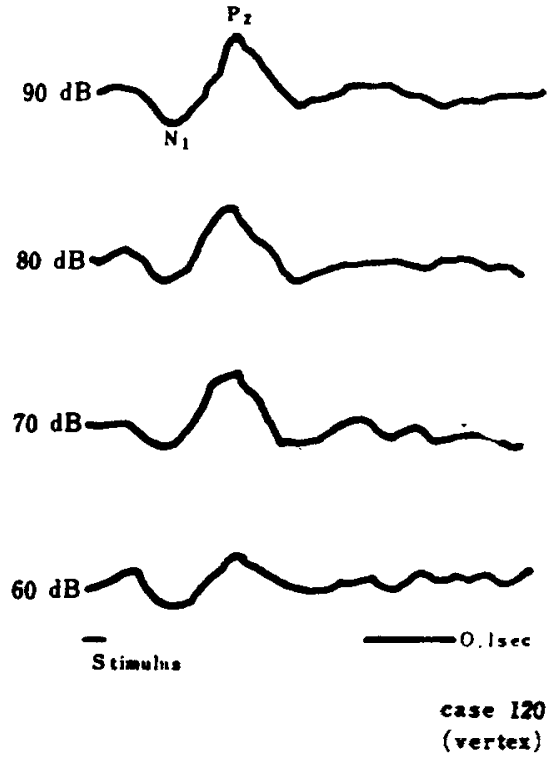

Fig. 8. Evoked potentials to tone pips of various intensity. Each trace is the average of $\mathbf{5 0}$ responses. Notice that $\mathbf{N}_{1} \cdot \mathbf{P}_{2}$ amplitude (peak latency, $N_{1}: 80$ msec; $P_{2}: 170$ msec) becomes larger according to the increase of stimulus intensity.

Kuwano, 1968), and the context effects on loudness judgments could be clearly recognized (Fig. 7 ).

Moreover, the experiment which Yasuda (née Kuwano), a member of our group, did (Kuwano, Shimokochi, \& Yamaguchi, 1969) will support this conclusion. This is an experiment where anchor effects were investigated using evoked potential (EVP) recorded

$(N=50)$

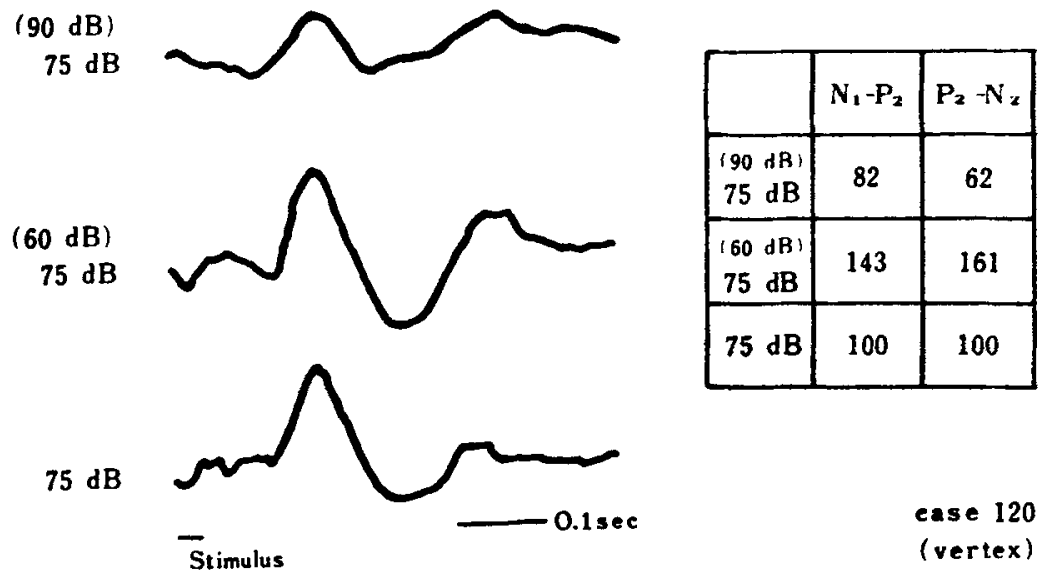

Fig. 9. Effects of anchor stimulus on the evoked potentials. Notice that the amplitude to a 75-dB tone is much larger when it follows a $60-\mathrm{dB}$ tone. Upper trace: EVP to 75-dB tone preceded by $90-\mathrm{dB}$ tone. Middle trace: EVP to $75-\mathrm{dB}$ tone preceded by 60-dB tone. Lower trace: Control; EVP when 75-dB tone was presented alone. 
to tones from the human scalp, especially the vertex. Pure tones of $1,000 \mathrm{~Hz} 75-\mathrm{dB}$ SPL were presented after 60- or 90-dB tones were presented several times and 50 responses to $75-\mathrm{dB}$ tones were averaged. Then peak-to-peak amplitude (N1-P2, peak latency 80 and $170 \mathrm{msec}$, respectively) of EVP to a 75-dB tone preceded by $60 \mathrm{~dB}$ was significantly much larger than that of the tone preceded by $90 \mathrm{~dB}$ (Fig. 9). Since the amplitude of these peaks becomes larger according to the increase of stimulus intensity, as seen in Fig. 8 , it may be conclusive that anchor effects exist in EVP as well.

We do not know if anchor effects occur which reflect a shift in perception. But since anchor effects were seen in three independent experiments, using category scale, RT, and EVP, we can conclude that anchor effects occur at the level of perception.

And, generally, in the investigation of some phenomena separating perception from responses, we would again like to emphasize the necessity of using various kinds of operations to make sure whether or not the phenomena depend on the operations. But since $R T$, used in Experiment 2, and EVP, used in Kuwano's experiment, are not sensory scales, there remain many unsolved problems in understanding how much anchor stimuli affect perception quantitatively.

\section{REFERENCES}

BELL R. A. \& BEVAN, W, Influence of anchors upon the operation of certain gestalt organizing principles. Journal of Experimental Psychology, 1968, 78, 670-678.

BEVAN, W., \& PRITCHARD, J. F. Effects of subliminal tones upon the judgment of loudness. Journal of Experimental Psychology, 1963, 66, 23-29.

BLACK, R. W., \& BEVAN, W. The effect of subliminal shock upon the judged intensity of weak shock. American Journal of Psychology, 1960, 73. 262-267.

BOARDMAN, W. K., \& GOLDSTONE, S. Effects of subliminal anchors upon judgment of size. Perceptual \& Motor Skills, 1962, 14, 457-482.

FILLENBAUM, S. Contextual effects in judgment as a function of restrictions in response language. American Journal of Psychology, 1963, 76, 103-109.

GOLDSTONE, S., GOLDFARB, J. STRONG, J., \& RUSSELL, J. Effect of subliminal shock upon judged intensity of weak shock. Perceptual \& Motor Skills, $1962,14,222$.

HARVEY, O. J., \& CAMPBELL, D. T. Judgments of weight as affected by adaptation range, adaptation duration, magnitude of unlabeled anchor, and judgmental language. Journal of Experimental Psychology, 1963, 65, 12-21.
HELSON, H. Adaptation-level theory. New York: Harper \& Row, 1964.

HELSON, H. \& KOZAKI, A. Anchor effects using numerical estimates of simple patterns. Perception \& Psychophysics, 1968,4, 163-164.

KUWANO, S., SHIMOKOCHI, M., \& YAMAGUCHI, Y. Auditory perception and evoked potential. Clinical Electroencephalography, 1969, 11, 144 .

MANABE, H., NAMBA, S., YOSHIKAWA, $T$.. \& KUWANO S. Automatic presentation of stimuli and processing of data in psychophysical experiments. Studies in the Humanities and Social Sciences, Osaka University, Faculty of General Education, 1969, 17, 71-79.

NAMBA S YOSHIKAWA, T., \& KUWANO, S. Context effects in loudness judgment. Japanese Journal of Psychology, 1968, 39, 191-199.

RAAB, D. H., \& GROSSBERG, M, Reaction time to changes in the intensity of white noise. Journal of Experimental Psychology, 1965, 69,609-612.

STEVENS, S. S. Adaptation-level vs. the relativity of judgment. American Joumal of Psy chology, 1958, 71, 633-646.

TACHIBANA, K, TASHIRO, T., MAKINO, T., \& NAMBA, S. On the allowance level of automobile phone. Proceedings of the 25th Annual Meeting of the Japanese Psychological Association, 1961.

YOSHIKAWA, T Effect of anchor stimulus on the judgment of loudness. Studies in the Humanities and Social Sciences, Osaka University, Faculty of General Education, 1968, 16, 77-105.

YOSHIKAWA $T$ \& NAMBA, S. Effects of anchor stimulus on the judgment of loudness. Reports of the autumn meeting of the Acoustical Society of Japan, 1966, 153.

(Accepted for publication June 22, $1971_{\circ}$ ) 